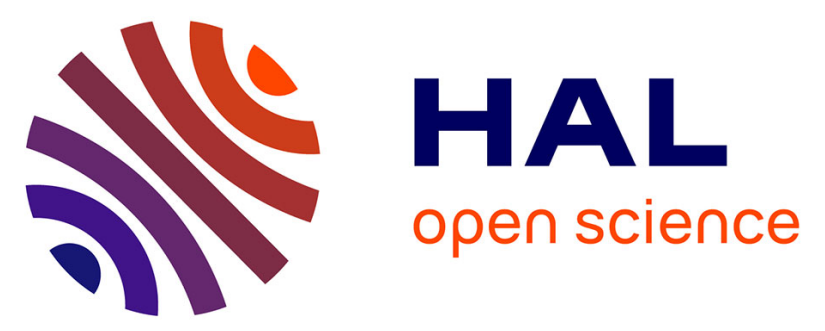

\title{
Identification du virus de rabougrissement buissonneux de la tomate (Tomato Bushy Stunt Virus) en Tunisie sur tomate, piment et aubergine: Quelques caractéristiques de la souche tunisienne
}

Christine Cherif, Didier Spire, M. A. Hasni

\section{To cite this version:}

Christine Cherif, Didier Spire, M. A. Hasni. Identification du virus de rabougrissement buissonneux de la tomate (Tomato Bushy Stunt Virus) en Tunisie sur tomate, piment et aubergine: Quelques caractéristiques de la souche tunisienne. Agronomie, 1983, 3 (7), pp.701-706. hal-00884562

\section{HAL Id: hal-00884562 https://hal.science/hal-00884562}

Submitted on 1 Jan 1983

HAL is a multi-disciplinary open access archive for the deposit and dissemination of scientific research documents, whether they are published or not. The documents may come from teaching and research institutions in France or abroad, or from public or private research centers.
L'archive ouverte pluridisciplinaire HAL, est destinée au dépôt et à la diffusion de documents scientifiques de niveau recherche, publiés ou non, émanant des établissements d'enseignement et de recherche français ou étrangers, des laboratoires publics ou privés. 


\title{
Identification du virus de rabougrissement buissonneux de la tomate (Tomato Bushy Stunt Virus) en Tunisie sur tomate, piment et aubergine : Quelques caractéristiques de la souche tunisienne
}

\author{
Christine CHERIF \& Didier SPIRE \\ avec la collaboration technique de M. A. HASNI \\ INRAT, Laboratoire de Virologie végétale, 2080 Ariana - Tunisie \\ I.N.R.A., Station de Pathologie végétale, Route de Saint-Cyr, F 78000 Versailles
}

RÉSUMÉ

Depuis 1979, nous avons observé à de nombreuses reprises le virus du rabougrissement buissonneux de la tomate (Tomato Bushy Stunt Virus: TBSV) dans le Nord-Est de la Tunisie dans des cultures de tomate (Lycopersicum esculentum L.), piment (Capsicum annuum L.) et aubergine (Solanum melongena L.). Le virus semble endémique dans la région et certaines années il provoque des dégâts importants. Les 3 isolats appartiennent à une même souche de TBSV, proche de la souche tomate décrite par SMITH en 1935. Parmi les variétés de tomate testées, 5 sont résistantes, 11 sensibles et 10 tolérantes. Aucune variété locale de piment n'est résistante. De nouveaux hôtes sensibles sont décrits ainsi qu'une méthode de purification sans ultracentrifugation.

Mots clés additionnels : Gamme d'hôtes, souches, sérologie, purification. characteristics of the Tunisian strain.

Tomato Bushy Stunt Virus (TBSV) was observed in different places in Tunisia, specially in the North-East region. TBSV was encountered on tomato plants (Lycopersicum esculentum) as well as pepper (Capsicum annuum) and eggplant (Solanum melongena). Serious reduction of yield was observed. After purification of the virus using a new method without ultracentrifugation, a strong antiserum was made. The host range of the virus was studied and new susceptible plants described. The three isolates of the virus belong to the same TBSV strain and were very similar to the original tomato strain described in 1935 by SMITH in Great Britain. Tomato varieties were tested for resistance. Five varieties were resistant, 11 susceptible and 10 tolerant. No local pepper varieties were resistant.

Additional key words : Host-range, strains, serology, purification.

\section{INTRODUCTION}

En Tunisie, les cultures maraîchères sont pour la plupart situées dans le Nord-Est du pays où la pluviométrie est supérieure à $400 \mathrm{~mm}$ par an et le long de la côte du Sahel (fig. 1). La tomate et le piment représentent $1 / 3$ de cette production et couvrent 30000 ha. Par contre, l'aubergine reste essentiellement une culture familiale. Ces 3 solanées maraîchères sont cultivées tout au long de l'année: en primeur, saison et arrière-saison. Au cours d'un inventaire des viroses qui attaquent ces cultures, nous avons identifié par voie biologique, d'une part sur tomate, la mosaique du tabac et la mosaiqque du concômbre, d'autre part sur piment, le virus $\mathrm{Y}$ de la pomme de terre, la mosaïque du tabac, la mosaique du concombre et la mosaïque de la luzerne (CHERIF, données non publiées).

Au printemps 1979, une grave maladie est observée au Cap Bon (fig. 1) sur tomate, piment et aubergine. Celle-ci se caractérise par un rabougrissement marqué des plantes ; les feuilles sont petites, cloquées, eniroulées et présentent des taches étoilées de couleur vert-clair ou jaune ; les feuilles plus anciennes ont des taches nécrotiques ; les fleurs ont tendance à avorter ; la production de fruits est extrêmement réduite et ceux-ci sont de petite taille (fig. 2 et 3 ). Chez l'aubergine, les rares fruits produits présentent des taches chlorotiques et des déformations. 


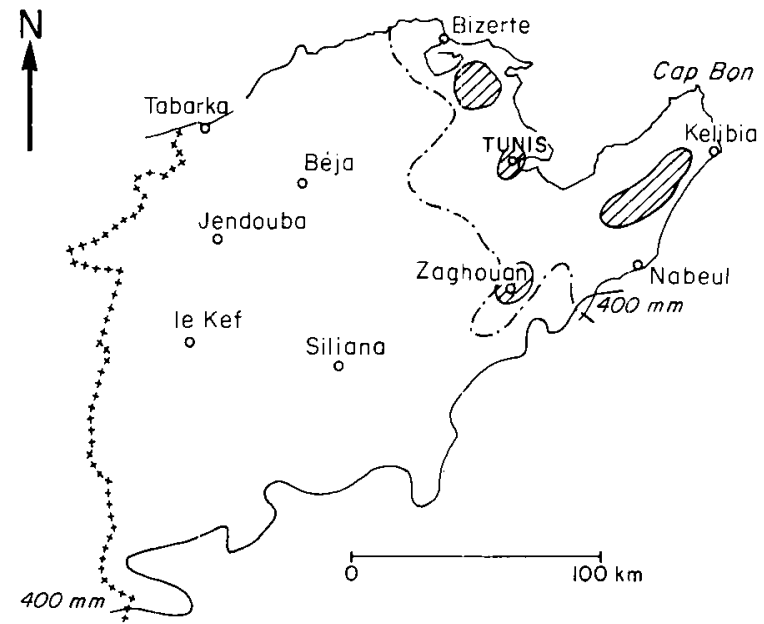

Figure 1. Tunisie septentrionale. Northern Tunisia.

Limite occidentale de la polyculture intensive. Western limits of intensive polyculture.

Régions infestées de TBSV. TBSV-infested regions.

En 1980 et 1981, cette même virose est également observée dans la région de Bizerte, Tunis et Zaghouan. Chez les variétés sensibles, jusqu'à 40 p. 100 des plantes extériorisent des symptômes à côté de variétés sans symptômes. Les différents tests décrits plus loin révèlent que les isolats obtenus des 3 espèces (tomate, piment, aubergine) appartiennent au Tomato Bushy Stunt Virus (TBSV).

Après une première mention au Maroc (FISCHER \& LOCKHART, 1977), c'est la deuxième fois que le TBSV est signalé au Sud de la Mćditerranée sur cultures de tomate et piment. Tout comme au Maroc et au Mexique (MARTINEZ et al., 1974), cette virose semble endémique dans le Nord-Est de la Tunisie. Certaines années les dégâts sont importants, tandis que d'autres années les symptômes se manifestent uniquement sur les rejets en fin de culture.

\section{MATÉRIEL ET MÉTHODES}

\section{A. Transmission mécanique}

Les cxtraits infecticux des 3 isolats sont obtenus en broyant les feuilles ou les plantes entières dans 3 volumes de tampon phosphate monopotassique-disodique $0,03 \mathrm{M}$, $\mathrm{pH} 7,3$ et 0,4 p. 100 de 2-mercaptoéthanol. Un minimum de 4 plantes par essai sont inoculées après adjonction de poudre de carborandum ( 600 mesh). Un témoin non inoculé est conservé dans chaque essai.

\section{B. Purification du virus}

Le virus est purifié selon la méthode de Hollings (1962), légèrement modifiée afin de l'adapter aux conditions de travail tunisienmes. Des feuilles ou des plantes entières infectées artificiellement par le TBSV (Nicotiana clevelandii Gray, tomate, piment ou Datura stramonium L.) sont broyées dans $2 \mathrm{ml} / \mathrm{g}$ de tissu de tampon phosphate monopotassique-disodique $0,05 \mathrm{M} \mathrm{pH} \mathrm{7,6}$ et 0,4 p. 100 de 2-mercaptoéthanol. On ajoute du n-butanol goutte à goutte jusqu'à

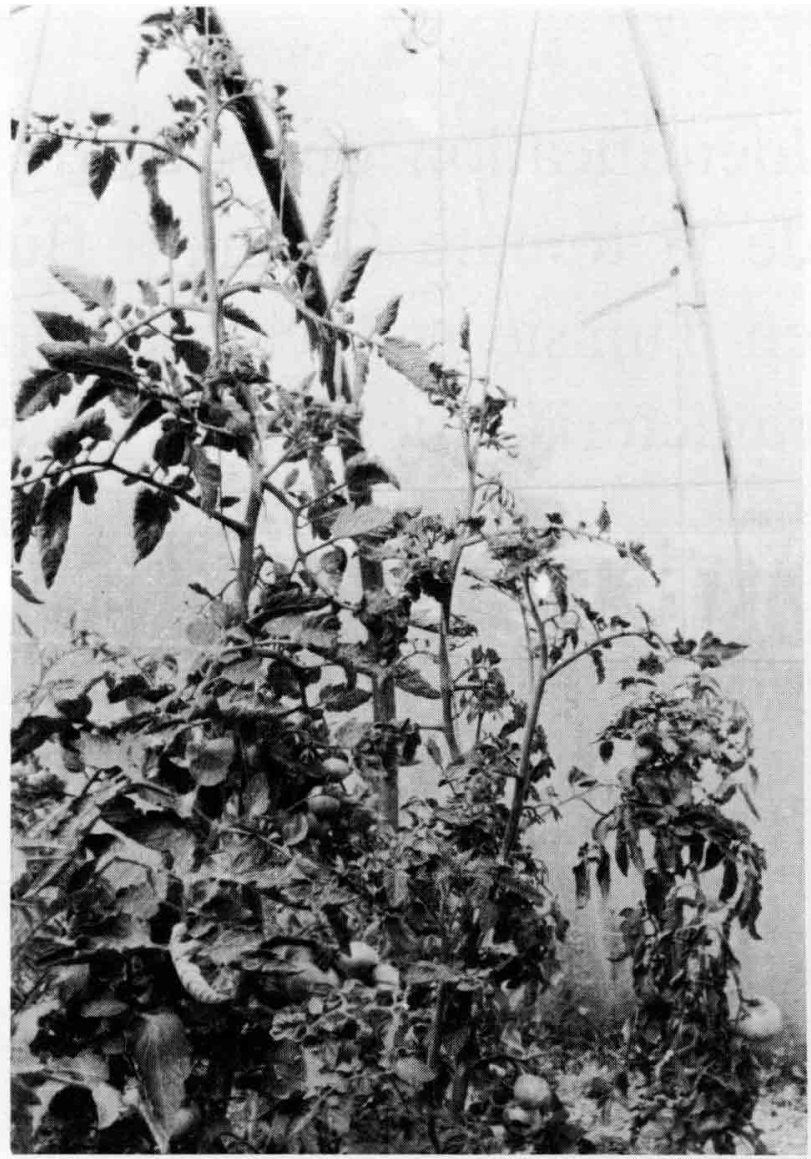

Figure 2

Symptômes de TBSV sur tomate.

TBSV symptoms on tomato.

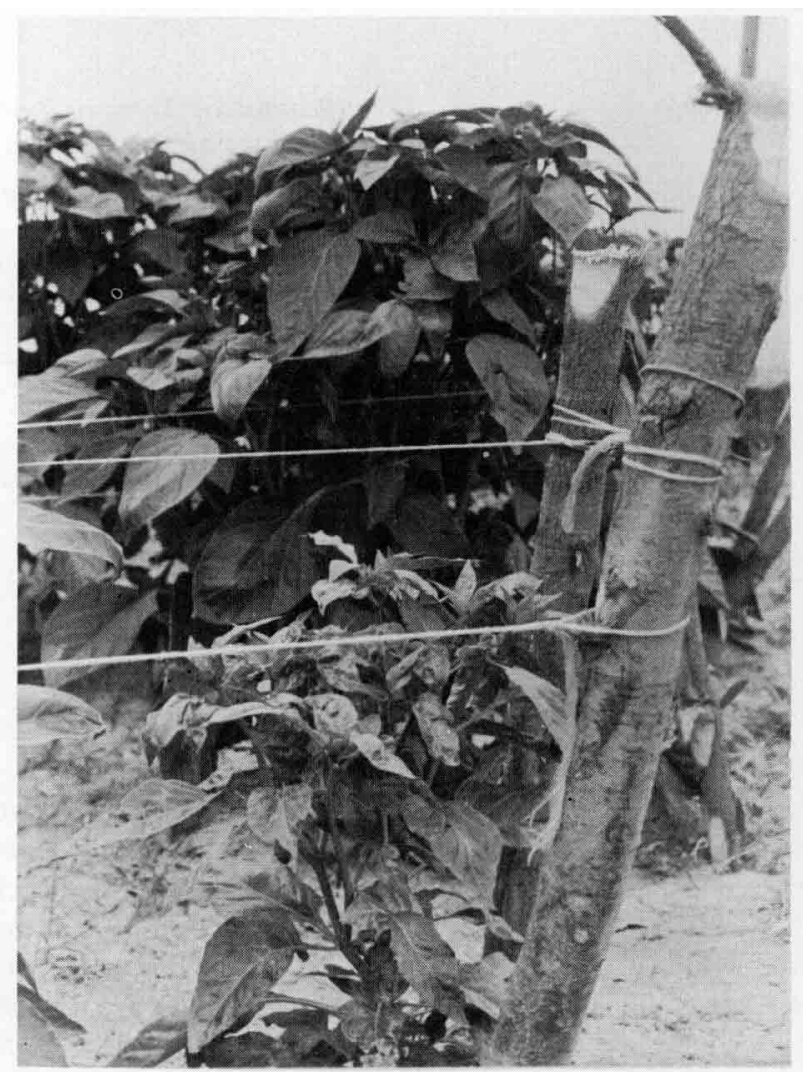

Figure 3

Symptômes de TBSV sur piment.

TBSV symproms on pepper. 
une concentration de 8,5 p. 100 . Le mélange est agité $30 \mathrm{mn}$ à $4{ }^{\circ} \mathrm{C}$, puis laissé au repos $24 \mathrm{~h}$ à $4{ }^{\circ} \mathrm{C}$. Le virus est ensuite concentré par 2 cycles de centrifugations différentielles, l'un de $20000 \mathrm{~g}$, l'autre de $47000 \mathrm{~g}(20000 \mathrm{t} / \mathrm{mn})$ pendant $3 \mathrm{~h}$, dans un rotor JA-20 d'une centrifugeuse Beckman J-21. Les culots sont repris dans un faible volume de tampon phosphate monopotassique-disodique $0,03 \mathrm{M}$ à $\mathrm{pH} 7,5$. Après homogénéisation et une centrifugation à basse vitesse, le surnageant est traité à la chaleur $(10 \mathrm{mn}$ dans un bain-marie à $70^{\circ} \mathrm{C}$ ) et refroidi immédiatement. Ce procédé permet d'éliminer le reste de protéines normales ayant précipité avec le virus.

Quant aux tombusvirus utilisés pour les tests sérologiques, ils sont purifiés selon la méthode récemment décrite par Koenig \& KUnZE (1982).

\section{Microscopie électronique}

Les extraits purifiés sont examinés en microscopie électronique (« Philips » EM 300) après coloration négative à l'acide phosphotungstique (2 p. 100 à pH 7).

\section{Tests sérologiques}

Ces tests sont réalisés en immunodiffusion dans une gélose à 0,6 p. 100 additionnée de 0,85 p. $100 \mathrm{de} \mathrm{NaCl}$ et 0,2 p. 100 de $\mathrm{NaN}_{3}$, le tout dissous dans un tampon $0,01 \mathrm{M}$ monopotassique-disodique $\mathrm{pH} 7,3$. Les antigènes sont déposés $5 \mathrm{~h}$ avant les antisérums.

Deux antisérums contre les extraits «piment" et " tomate» sont obtenus après 6 injections intramusculaires d'extrait purifié additionné d'adjuvant complet de Freund. Leur dilution limite se situe entre $1 / 256$ et $1 / 512$ en présence d'extraits purifiés de virus.

Les antisérums Petunia Asteroïd Mosaic Virus (PAMV) et Pelargonium Leaf Curl Virus (PLCV) ont été obtenus du Pr LOVISOLO de Turin; Artichoke Mottle Crinkle Virus (AMCV), TBSV-BS-3, Carnation Italian Ringspot Virus (CIRV), Cymbidium Ringspot Virus (CyRSV) du Pr MARTELLI de Bari et Eggplant Mottle Crinkle Virus (EMCV) du Pr MAKKOUK de Beyrouth.

\section{RÉSULTATS}

\section{A. Symptômes et gamme d'hôtes}

Les extraits virosés des 3 espèces (tomate, piment, aubergine) provoquent les mêmes réactions sur planteshôtes. Celles-ci sont présentées dans la tableau 1.

L'infection naturelle de 5 variétés de tomates, «Cal-j», «Cintra », «Fandango », « Noria » (F. 197), et « Vémone» a été suivic dans les champs et sous abris-plastique. A la suite de ces observations, des essais ont été réalisés afin de

TABLEAU 1

Gamme d'hôtes de la souche tunisienne de TBSV.

Host range of the TBSV Tunisian strain.

\begin{tabular}{|c|c|c|c|c|c|}
\hline Plantes inoculées & $\begin{array}{l}\text { Réactions } \\
\text { locales }\end{array}$ & $\begin{array}{l}\text { Réaction } \\
\text { généralisée }\end{array}$ & Plantes inoculées & $\begin{array}{l}\text { Réactions } \\
\text { locales }\end{array}$ & $\begin{array}{l}\text { Réaction } \\
\text { généralisée }\end{array}$ \\
\hline Atropa belladona L. & - & - & Nicotiana clevelandii Gray & II & $\mathrm{S}$ \\
\hline Capsicum annuum $\mathrm{L}$. & II & $\mathrm{S}$ & N. debneyi Domin & 11 & - \\
\hline C. chinense Jacq & II & $\mathrm{S}$ & N. glutinosa $\mathrm{L}$ & II & - \\
\hline C. flexuosum Sendt. & - & - & N. langsdorfii Wcinm & - & - \\
\hline \multirow{3}{*}{$\begin{array}{l}\text { C. frutescens L. } \\
\text { var. "Tabasco" }\end{array}$} & & & $N$. rustica $\mathrm{L}$. & 11 & - \\
\hline & 11 & - & & & \\
\hline & & & N. sanderae Sander. & - & - \\
\hline C. luteum Lam. & - & - & & & \\
\hline Celosia argentea $\mathrm{L}$. & 11 & $\mathrm{~S}$ & N. tabacum L. var.: & & \\
\hline Chenopodium amaranticolor & 11 & - & «Samsun » & 11 & - \\
\hline Coste et Reyn & & & «White Burlcy» & - & - \\
\hline & & & «Xanthi $»$ & - & - \\
\hline C. quinoa Willd. & 11 & - & «Burlcy BO-5» & 11 & - \\
\hline Cucumis sativus $\mathrm{L}$. & 11 & - & Ocimum basilicum $\mathrm{L}$. & Il & - \\
\hline Datura inoxia Mill. & II & - & Pelargonium zonale $\mathbf{L}$. & II & - \\
\hline D. metel $\mathrm{L}$. & 11 & $S$ & Petunia hybrida Vilm. & II & - \\
\hline D. stramonium $\mathbf{L}$. & II & $\mathrm{S}$ & Phaseolus vulgaris L. «Pinto» & $\$ 1$ & - \\
\hline Gomphrena globosa $\mathrm{L}$. & II & - & Physalis angulata $\mathrm{L}$. & - & SS \\
\hline Hyoscyamus albus L. & 11 & - & P. floridana Rydb & 11 & - \\
\hline H. niger $\mathrm{L}$. & - & $S$ & P. peruviana $\mathrm{L}$. & - & - \\
\hline Lavatera trimestris $\mathrm{L}$. & 11 & - & Senecio cruentus DC. & II & - \\
\hline Lycium afrum $\mathrm{L}$. & & - & Solanum capsicastrum LK. & - & SS \\
\hline Lycopersicum cerasiforme & II & $\mathrm{S}$ & S. dulcamara $\mathrm{L}$. & - & SS \\
\hline (Dun.) Voss. & & & S. melongena $\mathrm{L}$. & $\|$ & $\mathrm{S}$ \\
\hline L. esculentum Mill. & II & $\mathrm{S}$ & S. nigrum L. & - & $S$ \\
\hline L. hirsutum Mill. & - & - & S. sodomeum $\mathrm{L}$. & - & - \\
\hline L. peruvianum Mill. & - & $\mathrm{S}$ & $S$. tuberosum L. var. "Bintje» & II & - \\
\hline L. pimpinellifolium Mill. & - & - & Tropaeolum majus $\mathrm{L}$. & II & - \\
\hline Nicotiana acuminata Hook & - & - & Vigna sinensis Savi & II & - \\
\hline N. bigelovii (Tor.) Watson & 11 & $\mathrm{~S}$ & Zinnia elegans Jacq. & II & - \\
\hline
\end{tabular}

II : Lésions locales ; local lesions.

$\mathrm{S}$ : Symptômes généralisés visibles; visible systemic symptoms.

SS : Infection sans symptômes; infection without symptoms.

- : Pas d'infection; no infection. 
tester la sensibilité de variétés et hybrides utilisés en Tunisic.

- 9 variétés sont infectées artificiellement par inoculation mécanique et manifestent des symptômes caractéristiques de l'infection par le TBSV: «Cal-j», «Canatella , «Epona », «GC-204», « Montfavet H63-5 », « Noria » (F197), « Porphyre », « Severiani », « Vémone».

- 10 variétés sont infectées artificicllement par inoculation mécanique et ne manifestent pas de symptômes. Cependant le virus peut être mis en évidence au sommet de la plante par rétroinoculation : «Early PAK 7 », « Florida MH1 ", "Lucy ", " Momor », " Monita », " Ohio MR 12 ", «Roma », «Tropic », «Ventura », «WN-63».

- Enfin 5 variétés ne sont infectées ni naturellement ni artificiellement et le virus ne peut être mis en évidence au sommet de la plante par rétroinoculation: "Jolimac», « Marmande », « Mobaci », « Motelle », « Picrsol».

Les variétés «Cintra» et «Fandango » n'ont pu être testées faute de semences. Cinq variétés locales de piment sont testées par inoculation mécanique; elles sont toutes sensibles au TBSV: «le piment d'Algérie", "Bcldi", d'« Hirat », «Korba » ct « Meski ».

\section{B. Purification et microscopie électronique}

Les extraits ainsi purifiés, examinés au microscope électronique permettent l'observation de particules virales parasphériques de $30 \mathrm{~nm}$ de diamètre (fig. 4).

\section{Sérologie}

Lorsque les extraits correspondant aux isolats des 3 espèces, tomate $(T)$, piment $(P)$, aubergine $(A)$ sont placés dans des réservoirs adjacents, on observe une ligne de précipitation continue, sans formation d'éperon, quel que soit l'antisérum utilisé. Les 3 isolats appartiennent à une seule et même souche de TBSV.

Cette souche comparéc à d'autres tombusvirus (tabl. 2) donne les résultats suivants :

Avec la souche tomate (BS-3), le précipité obtenu forme une ligne continue. La souche tunisienne est donc très proche sinon identifique à la BS-3 (fig. 5).

Par contre avec AMCV, PLCV, PAMV, EMCV et CIRV on observe un précipité avec formation d'éperons. Bien que reliés sérologiquement, ces virus sont différents comme l'indiquent la formation d'éperon, la comparaison des titres des antisérums en plus de leur gamme d'hôtes; (LOvisolo, 1957 ; Hollings, 1962 ; Hollings \& STONE, 1965 ; MaRTELLI, 1965 ; Hollings et al., 1970 ; CHERIF, 1981 ; MARTELLI, 1981 ; MAKKOUK et al., 1981).

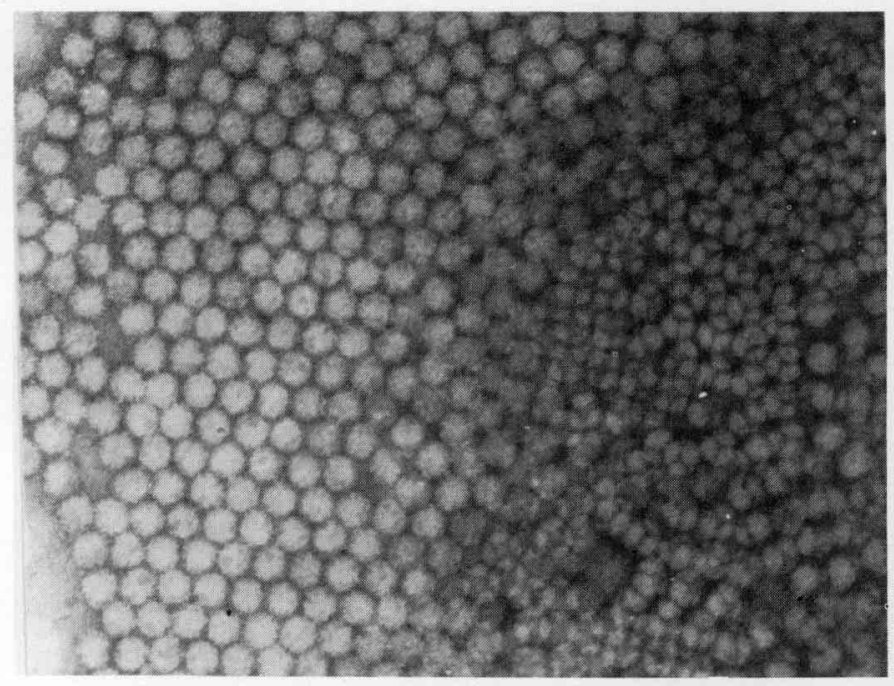

Figure 4

Aspect au microscope électronique d'un extrait purifié de la souche tunisienne de TBSV $(\times 135000)$.

Negatively stained particles of the Tunisian strain of TBSV.

Avec le Cymbidium Ringspot (CyRSV) aucune réaction n'est observée, ce qui confirme les résultats de HoLlings et al. (1977).

\section{DISCUSSION}

Les propriétés biophysiques, morphologiques et sérologiques du virus isolé en Tunisie sont caractéristiques des tombusvirus. Sa gamme d'hôtes et ses propriétés sérologiques le rapprochent de la souche type de TBSV décrite par SMITH (1935). Cependant pour la première fois, une même souche de TBSV infecte en condition naturclle 3 espèces de solanées : la tomate, le piment et l'aubergine, sur lesquelles elle provoque des symptômes caractéristiques des tombusvirus. Les souches « tomate » décrites dans le monde n'infectent que cette espèce (SMITH, 1935 ; PONTIS et al., 1968 ; MARTELLI et al., 1972 ; MARTINEZ et al., 1974 ; FISCHER \& LOCKHART, 1977 ; BORGES et al., 1979).

Les tests biologiques révèlent qu'en plus des hôtes sensibles propres aux autres souches "tomate» de ce virus, de nouveaux hôtes sont mis en évidence (tabl. 1). Il s'agit notamment de :

Celosia argentea L.: la formation de petites taches nécrotiques circulaires de couleur rouge généralisées à toute la plante, et nanisme marqué.

TABLEAU 2

Titres homologues et hétérologues de deux antisérums TBSV vis-à-vis d'extraits de différents Tombusvirus, en immunodiffusion. Homologous and heterologous titres of two TBSV antisera facing some Tombusvirus extracts.

\begin{tabular}{|c|c|c|c|c|c|c|c|}
\hline Antigc̀nes $(*)$ & TBSV BS-3 & TBSV-Tun. & $\mathrm{AMCV}$ & PLCV & PAMV & EMCV & CIRV \\
\hline \multicolumn{8}{|l|}{ Antisérums } \\
\hline TBSV-Tun. & 128 & 128 & 32 & 32 & 16 & 8 & 8 \\
\hline S.D.I. $\left(^{*}\right)$ & 0 & 0 & 2 & 2 & 3 & 4 & 4 \\
\hline
\end{tabular}

(*) Serological Differential Index. 


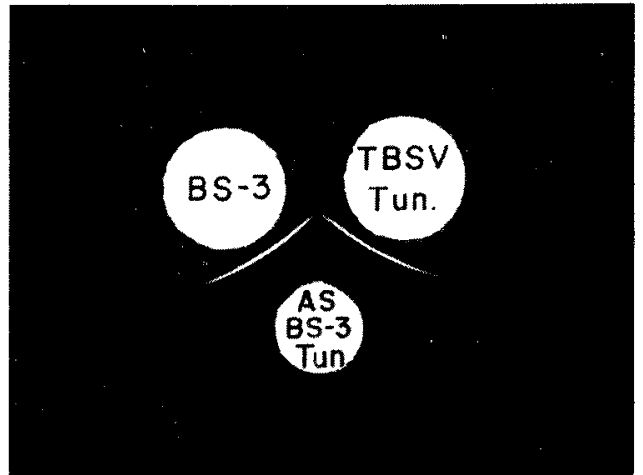

Figure 5

Comparaison sérologique des souches tunisiennes et BS-3 de TBSV.

Hyoscyamus niger L.: mosaïue jaune et nanisme si prononcé que les plantes ne dépassent pas $5 \mathrm{~cm}$ et restent en forme de rosette.

Lycopersicum cerasiforme (Dun) Voss: chlorose et nanisme.

Nicotiana bigelovii (Torrey) Watson: grandes lésions locales chlorotiques suivies de taches jaunes et flétrissement 10 à $15 \mathrm{j}$. après l'inoculation.

Datura stramonium L. extériorise les mêmes symptômes marqués de mosaïque et déformations avec les 3 souches «tomate» (tabl. 3), cependant la souche marocaine ne s'y généralise que chez les plantes très jeunes (FISCHER \& LOCKHART, 1977). La souche marocaine provoque des lésions locales chlorotiques sur D. metel L., mais ne s'y généralise pas contrairement aux souches argentine (PoNTIS

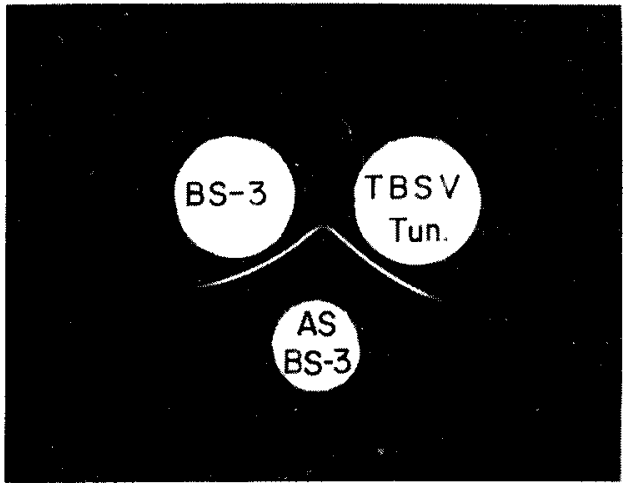

Serological comparison of the Tunisian and BS-3 strains of TBSV.

et al., 1968) et tunisienne qui produisent une mosaïque plane. La souche anglaise de TBSV (SMITH, 1935) cause des lésions locales sur Nicotiana langsdorfii Weinm. et N. tabacum L. variété «White Burley», et les souches marocaines en provoquent également sur la variété «Xanthi », mais pas la souche tunisienne (tabl. 3).

Malgré les quelques différences énumérées ci-dessus, les souches «tomate" marocaine (FISCHER \& LOCKHART, 1977) et tunisienne se rapprochent de la souche type de TBSV. Ces observations sont confirmées par les réactions de précipitation en immunodiffusion : la souche tunisienne semble tout à fait identique à la souche BS-3 de STEERE (1953), elle-même dérivée de celle de SMITH (1935).

Par contre, la souche "piment» ou Moroccan Pepper Virus (MPV) se généralise chez différents Chenopodium

\section{TABLEAU 3}

Comparaison des symptômes provoqués sur diverses espèces par les souches de TBSV qui attaquent les Solanacées maraîchères. Comparison between TBSV strains infecting solanaceous vegetable crops.

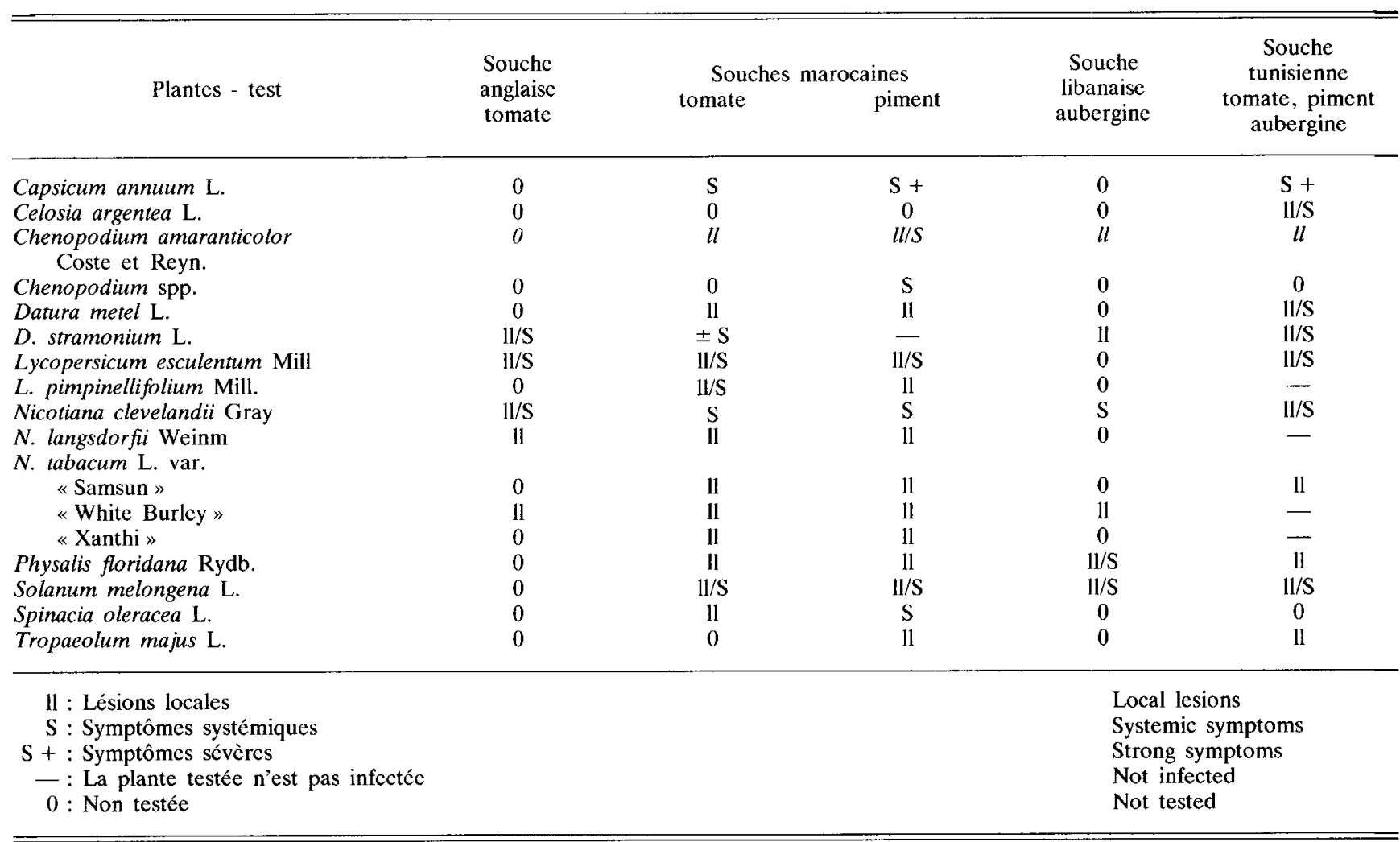


spp. ainsi que Spinacia oleracea L., mais pas chez Datura stramonium L. ni $D$. metel L. Ce virus est relié sérologiquement aux autres tombusvirus mais n'est identique à aucun d'entre eux (FISCHER \& LOCKHART, 1977).

Au Liban, MAKKOUK et al., (1981) ont décrit un tombusvirus sur aubergine: Eggplant Mottle Crinkle Virus (EMCV) dont les symptômes au champ sont semblables à ceux observés en Tunisie sur cette espèce. Cependant la gamme d'hôtes diffère : l'EMCV se généralise chez Physalis floridana Rybd. alors que la souche tunisienne n'y provoque que des lésions locales nécrotiques et n'infecte pas Datura stramonium L. De plus, comme le montre le tableau 2 et les travaux de MAKkoUK et al. (1981), ce virus, bien que relié sérologiquement à la souche type et à la souche tunisienne, en est cependant éloigné. L'étude de l'épidémiologie de ce tombusvirus en Tunisie est en cours.

Reçu le 5 mai 1982. Accepté le 25 mars 1983.

\section{REMERCIEMENTS}

Nous remercions les professeurs O. Lovisolo (laboratorio di Fitovirologia applicata, Turin, Italie), K. M. MaKKOUK (Université américainc, Beyrouth, Liban), G. P. MARTELli et M. Russo (Istituto di patologia vegetale, Bari, Italie), pour les dons de sérums et d'antigènes, Mme Michon (Station de Pathologie végétale, Versailles) pour les obscrvations en microscopie électronique, $\mathrm{Mr}$. N. Hamza (INRAT, Ariana, Tunisie) pour les dons de semences.

\section{RÉFÉRENCES BIBLIOGRAPHIQUES}

Borges M. L., Sequeira J. C., Louro D., 1979. Apparition au Portugal du Virus du Rabougrissement Buissonneux de la Tomate (Tomato Bushy Stunt Virus). Gamme d'hôtes, morphologic et localisation dans les cellules de piment. Phytopathol. mediter., 18, 118-122. (en portugais)

Cherif C., 1981. Contribution à la connaissance du Virus $d u$ Rabougrissement Buissonneux de la Tomate (Tomato Bushy Stunt Virus) en Tunisie. Thèse $3^{c}$ cycle : Biol.-Physiol. vćg., Paris 6, 85 p.

Fischer H. U., Lockhart B. E. L., 1977. Identification and comparison of two isolates of Tomato Bushy Stunt Virus from pepper and tomato in Morocco. Phytopathology, 67, 1352-1355.

Hollings M., 1962. Studies of Pelargonium leaf curl virus. I. Host range, transmission and properties in vitro. Ann. appl. Biol., 50, 189-202.

Hollings M., Stone O. M., 1965. Studics of Pelargonium leaf curl virus. II. Relationships to tomato bushy stunt and other viruses. Ann. appl. Biol., 56, 87-98.

Hollings M., Stone O. M., Boutell G. C., 1970. Carnation Italian Ringspot Virus. Ann. appl. Biol., 65, 299-309.

Hollings M., Stone O. M., Barton R. J., 1977. Pathology, soil transmission and characterization of Cymbidium ringspot, a virus from Cymbidium orchids and whitc clover (Trifolium repens) Ann. appl. Biol., 85, 233-248.

Koenig R., Kunze L., 1982. Identification of tumbusvirus isolates from cherry in southern Germany as Petunia asteroid mosaic virus. Phytopath. Z., 103, 361-368.
Lovisolo O., 1957. Petunia : nuovo ospite naturale del virus del rachitismo cespuglioso del pomodoro. Boll. staz. Pat. veg., 14 (3), 103-119.

Makkouk K. M., Koenig R., Leseman D. E., 1981. Characterization of a tombusvirus isolated from eggplant. Phytopathology, 71, 572-577.

Martelli G. P., 1965. L'arricciamento maculato del carciofo (Cynara scolymus). Phytopathol. medit., 4, 58-60.

Martelli G. P., 1981. Tombusviruses - in Handbook of Plant Virus infections and Comparative Diagnosis pp. 61-90. E. Kurstak (ed). -- Elsevier/North-Holland Biomedical Press.

Martelli G. P., Quaquarelli A., Russo M., 1971. Tomato Bushy Stunt Virus in Descriptions of Plant Viruses $-n^{\circ} 69$. CMI/AAB Kew, Surrey England 4.

Martinez A. J., Galindo J. A., Rodriguez M. R., 1974. Estudio sobre la enfermedad del "pinto" del tomate (Lycopersicum esculentum Mill) en la region de Actopan. Hgo. Agrociencia, 18, 71-78.

Pontis R. E., Garcia O., Feldman J. M., 1968. Tomato Bushy Stunt Virus on tomato crops in Argentina. Plant. Dis. Rep., 52, 676-677.

Smith K. M., 1935. A new disease of the tomato. Ann. appl. Biol., 22, 731-741.

Steere R. L., 1953. Strains of tomato bushy stunt virus. Phytopathology, 43, 485. 